\title{
Image Enhancement Using Vector Quantisation Based Interpolation
}

\author{
W. Paul Cockshott, Sumitha L. Balasuriya, Irwan Prasetya Gunawan, \\ and J. Paul Siebert \\ University of Glasgow, Computing Science Department \\ 17 Lilybank Gardens, Glasgow G12 8QQ \\ \{wpc, sumitha, ipguna, psiebert\}@dcs.gla.ac.uk
}

\begin{abstract}
We present a novel method of image expansion using vector quantisation. The algorithm is inspired by fractal coding and uses a statistical model of the relationship between details at different scales of the image to interpolate detail at one octave above the highest spatial frequency in the original image. Our method aims at overcoming the drawbacks associated with traditional approaches such as pixel interpolation, which smoothes the scaled-up images, or fractal coding, which bears high computational cost and has limited use due to patent restrictions. The proposed method is able to regenerate plausible image detail that was irretrievable when traditional approaches are used. The vector quantisation-based method outperforms conventional approaches in terms of both objective and subjective evaluations.
\end{abstract}

\section{Introduction}

Digital cinema sequences can be captured at a number of different resolutions, for example $2 \mathrm{~K}$ pixels across or $4 \mathrm{~K}$ pixels across. The cameras used for high resolutions are expensive and the data files they produce are large. Because of this, studios may chose to capture some sequences at lower resolution and others at high resolution. The different resolution sequences are later merged during post production. The merger requires that some form of image expansion be performed on the lower resolution sequences. In this paper we present a new method of doing the image expansion that has some advantages over the orthodox interpolation methods.

The paper is organised as follows. Section 2 will review some of the existing techniques of image expansion and highlight their shortcomings. In Section 3, we will describe the proposed algorithm in details including the process of training the algorithm, constructing the library used in it, and producing as well as enhancing the expanded image using the algorithm. Section 4 contains our experimental results in which our proposed method is evaluated. The paper concludes in Section 5. 


\section{Background}

In the traditional pixel interpolation method, new pixels are generated in the scaled-up image; however, there is no information pertaining to what these pixels should contain, other than interpolation of the original pixels by some polynomial function. Because the polynomial function works over a neighbourhood in the original, smaller image, the scaled-up image will contain less energy at the highest spatial frequencies than the original, making it looks smoother.

An alternative approach through fractal encoding, originally reported by Barnsley [1], allows rescaled images to contain new high frequency information. Fractal encoding takes advantage of the self similarity across scales of natural scenes. A fractal code for an image consists of a set of contractive affine maps from the image, onto the image. Taken as a whole, these maps compose a collage such that each pixel is mapped onto by at least one such map. The maps operate both in the spatial and the luminance domain. In the luminance domain they specify a target pixel $p$ by an equation of the form $p=a+b q$ where $q$ is the mean brightness of a downsample region of source pixels. In the spatial domain they specify the coordinates of the source pixels supporting $q$ as the result of rotation, scaling and translation operations on the coordinates of the destination pixels.

The image is regenerated from the codes by iterated application of the affine maps. The iteration process has an attractor that is the output image. If the maps have been well chosen this attractor approximates well to a chosen input image.

A particular fractal code might specify each $4 \times 4$ rectangle within a $256 \times 256$ pixel output image in terms of a contractive map on some $8 \times 8$ rectangle at some other point in the image. As the iteration proceeds higher and higher frequency information is built up. If we start from a uniform grey image, the first iteration will generate detail at a spatial frequency of 8 pixels. After one iteration source blocks of 8 pixels across will contain up to one spatial wave. After the second interpolation these waves will have been shifted up in frequency to 4 pixels across. Each iteration adds detail one octave higher until the Nyquist limit of the output image is reached: 128 spatial cycles in this case.

It is evident that if we specify the contractive mappings relative to the scale of the whole image rather than in terms of pixels, then the same set of mappings could be used to generate a $512 \times 512$ pixel output image. In this case the contractive mappings would shrink 16 pixel blocks to 8 pixel blocks. After an additional round of iteration the 512 pixel output image will contain spatial frequencies up to 256 cycles.

Fractal codes can thus be used to expand an image, generating new and higher spatial frequencies in the process. Although the additional detail that is added by this process can not have been available in the source image it nevertheless 'looks plausible' because the 'new' details are scaled down versions of details that were present in the original picture (see Figure 1). The search process used in a fractal encoder scans a half sized copy of the original image to find a match for each small block in the original image. In fractal enhancement the small blocks are then replaced by their full sized corresponding blocks. The detail enhancement comes because there is a systematic relationship between the low frequency and high frequency information within blocks. This allows high frequency information in a larger block to be plausibly substituted into a smaller block when the latter is enlarged.

Fractal codes however suffer from two serious obstacles to their widespread adoption: the encoding algorithm is slow and their general use is blocked by patent restrictions. In 


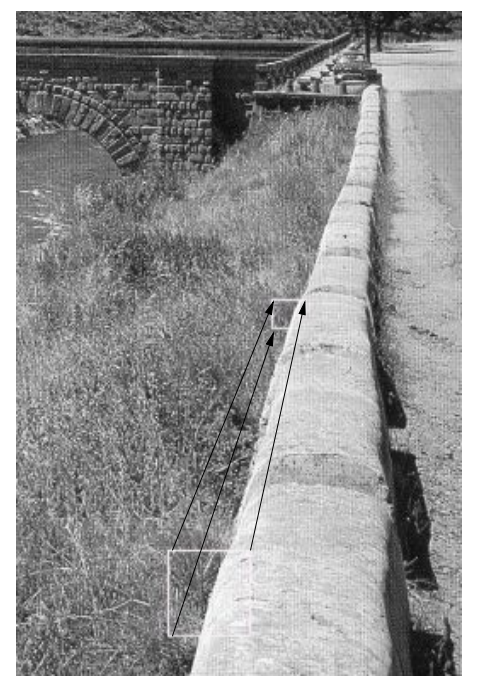

Figure 1: Illustration of how shrinking is used to fill in detail in fractal enhancement.

this paper we present an alternative approach that learns lessons from fractal coding but avoids these difficulties. Instead of using fractals we use vector quantisation to enhance the detail of an image.

\section{Proposed method}

The key idea of our approach is that because there is a systematic relationship between low and high frequency information within a neighbourhood, it should be possible for a machine learning algorithm to discover what this relationship is and exploit this knowledge when enhancing an image. We use vector quantisation to categorise areas of the image at different scales, learn the systematic relationship between the coding of corresponding areas at varying scales, and then use this information to extrapolate a more detailed image. The entire process works by

1. Running a training algorithm to learn the cross-scale structure relations in example pictures. In the experiments here two images were used; one from the 'face' sequence and one from the outside 'trees' sequence.

2. Using this information to automatically construct a new image enhancing program.

3. Applying the enhancing program to digital cine images to generate new images at twice the resolution.

\subsection{The Training Algorithm}

The aim of the training algorithm is to learn what high frequency detail is likely to be associated with the low frequency features at a given point in an image. Given an image $I$ we construct a half sized version of the image $I_{0.5}$ and expand this to form a new blurred image $I_{b}$ which is the original size, by using linear interpolation. We now form a difference image $I_{d}=I-I_{b}$ which contains only the high frequency details. 
It is clear that we have a genetive association between position $I_{0.5}[x, y]$ and the four pixel block $G_{x, y}=\left\{I_{d}[2 x, 2 y], I_{d}[2 x+1,2 y], I_{d}[2 x, 2 y+1], I_{d}[2 x+1,2 y+1]\right\}$. We aim to categorise the regions around each position in $I_{0.5}[x, y]$, categorise the corresponding blocks $G_{x, y}$ and learn the associations between these categories.

\subsubsection{Categorising the Upper Layer}

Associate with each pixel $p \in I_{0.5}$ a neighbourhood $\square$ and compute the differences between $p$ and its neighbours. These define a 4 element vector. Using the algorithm given in [6] construct a vector quantisation codebook $B_{1}$ for these features. Assume that the code book has $n$ entries.

\subsubsection{Categorising the Lower Layer}

Use the same vector quantisation algorithm to construct a second vector quantisation codebook $B_{2}$ for the set of vectors $G_{x, y}$. Assume that the code book again has $n$ entries.

\subsubsection{Learning the Association}

Encode the neighbourhoods around each pixel $p \in I_{0.5}$ with $B_{1}$ to yield an encoded image $E_{0.5}$. Encode each $G_{x, y}$ associated with each pixel $p \in I_{0.5}$ with $B_{2}$ to yield an encoded image $E_{b}$. The entries in both the encoded images are indexed into the respective codebooks.

Construct an $n \times n$ frequency table $F$ that counts how frequently each code from $B_{1}$ is associated with each code from $B_{2}$. Finally convert the frequency table to a conditional probability table by dividing by the number of observations.

\subsection{The Program Generator}

The aim of the program generator is to take the tables $B_{1}, B_{2}, F$ and use them to generate pascal libraries that can be used to index and predict detail in subsequent images. The process is analogous to the way Lex [5] constructs scanner tables in $\mathrm{C}$ from a regular grammar.

Two optimisations are performed prior to outputting the tables:

1. Table $F$ is converted from a conditional probability table to a table encoding the cumulative probability of each entry in $B_{2}$ being associated with and entry in $B_{1}$.

2. Hierarchical Vector Quantisation [2] indices are constructed for the two codebooks to enable future encoding to be of $\mathbf{O}(4)$ rather than $\mathbf{O}(\mathrm{n})$.

\subsection{The Enhancement Algorithm}

The enhancement program has the library produced above linked to it. The aim of the program is to read in an image $J$ and produce an image $J_{2}$ of twice the size with enhanced detail. The algorithm is described by Algorithm 1 .

Once this process has been completed for each pixel in $J$ the image $J_{d}$ contains details whose spatial frequency is one octave higher than those that are represented in $J_{b}$. Each 


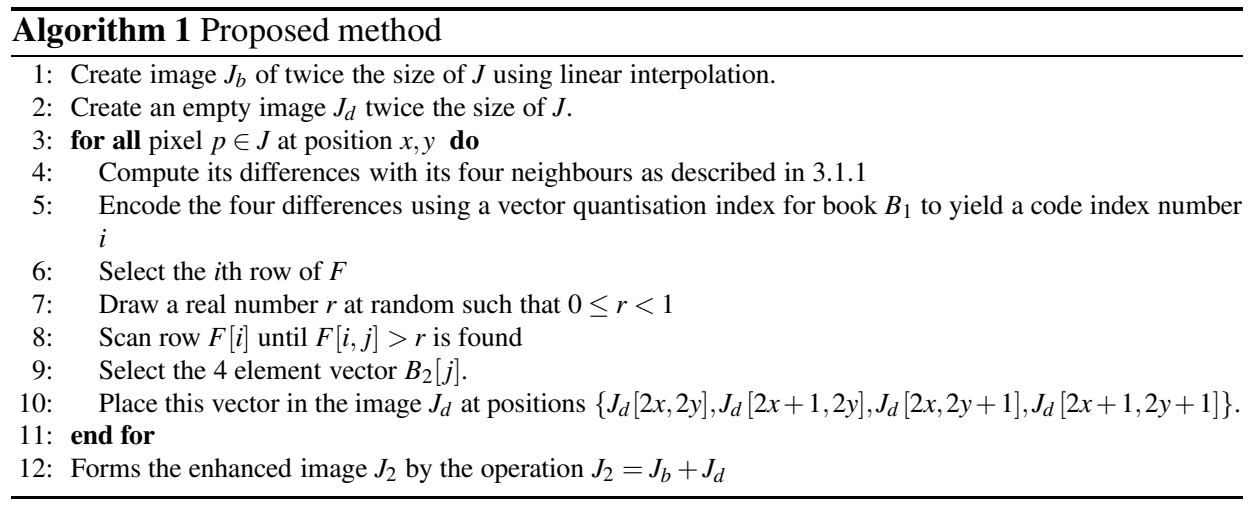

detail occurs with the same probability with respect to the categorisation of localities in $J$ as details occurred in $I_{d}$ with respect to the categorisation of localities in $I_{0.5}$.

\section{Results}

Our experiments were conducted on $1920 \times 1080$ video frames in the DPX image format captured by a Thompson Viper D-Cinema video camera. The pixels were in 10 bit logarithmic format. Image expansion using our system was compared to conventional bilinear and B-spline interpolation techniques. The experimental procedure can be described as follows:

1. The enhancement system was trained on a $1920 \times 1080$ DPX frame from an outdoor sequence. The test images used were a studio frame (Figure 2), a later frame from the outdoor sequence from which original training frame had been selected (Figure 4), and a train sequence (Figure 6). The training and test frame from this sequence had different zoom settings, the training frame having had a higher zoom factor than the second test frame.

2. The test images were downsampled using bilinear interpolation to $960 \times 540$ and output in DPX format.

3. Image expansion to double the original resolution of $1920 \times 1080$ was performed using our algorithm, bilinear and B-spline interpolations.

4. Original and expanded DPX video frames were compared subjectively based on perceived detail in image patches. The quality of reproduction was also evaluated objectively using several image quality metrics described below.

We used several methods of assessment to evaluate the quality of the reconstructed images. First, a traditional measure based on Peak Signal-to-Noise Ratio (PSNR) [7] was calculated. In this paper, the PSNR was calculated on the 10-bit logarithmic representation of pixel values. This metric is very practical and easy to compute, however critic says that it does not always correlate well with the quality perceived by human users [3]. An alternative using a modified version of the PSNR based on perceived visibility of error, namely Weighted Peak Signal-to-Noise Ratio (WPSNR) [8], was also computed. In this metric, error on textured area would be given less weighting factor than that on flat surface. 


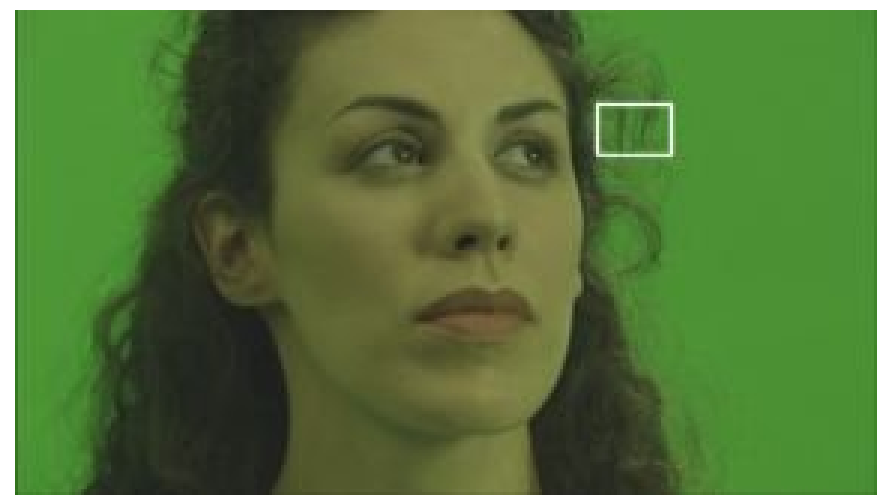

Figure 2: Reduced scale colour image from original DPX digital cine frame from studio sequence 'face'. Relatively soft focus is used with a moving subject. Box indicates where detail is shown in Figure 3. Note that this and all following images are uncorrected log colour space.

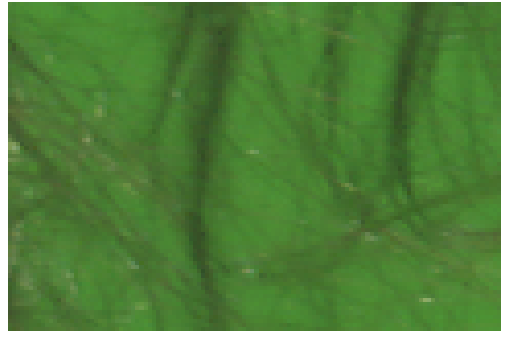

(a) Original

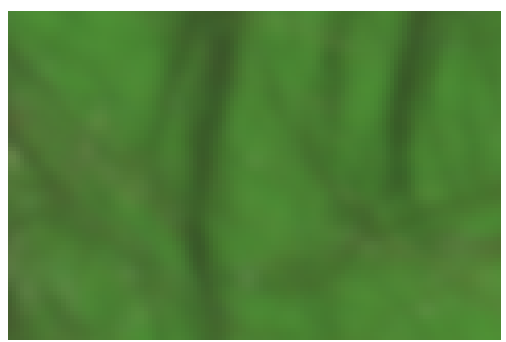

(c) B-spline

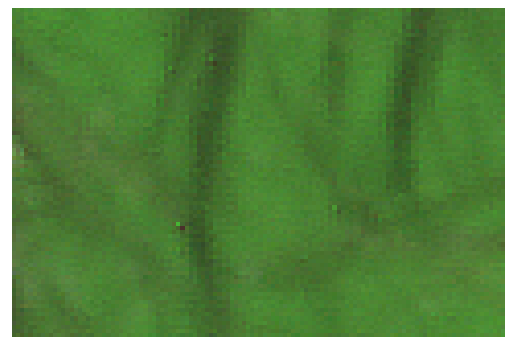

(b) Our algorithm

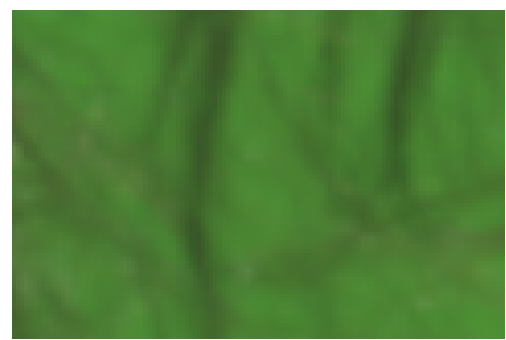

(d) Bilinear

Figure 3: A region with high frequency detail from original DPX video frame from the 'face' sequence (a) and the corresponding region generated by expansion of the half-resolution video frame by our algorithm (b), as well as by B-spline interpolation (c) and bilinear interpolation (d).

Since image expansion algorithms usually introduce blur artifacts, a quality metric [4] capable of detecting and measuring the degree of blurriness on the degraded image was used. This metric computes features extracted from the frequency domain through two-dimensional Discrete Fourier Transfrom (DFT) computation over a localised area on the gradient image. In an image contaminated by blurring distortion, some frequency components appear attenuated when compared to the corresponding set of components on the original image. Blurriness detection can be done by analysing the decay in the 


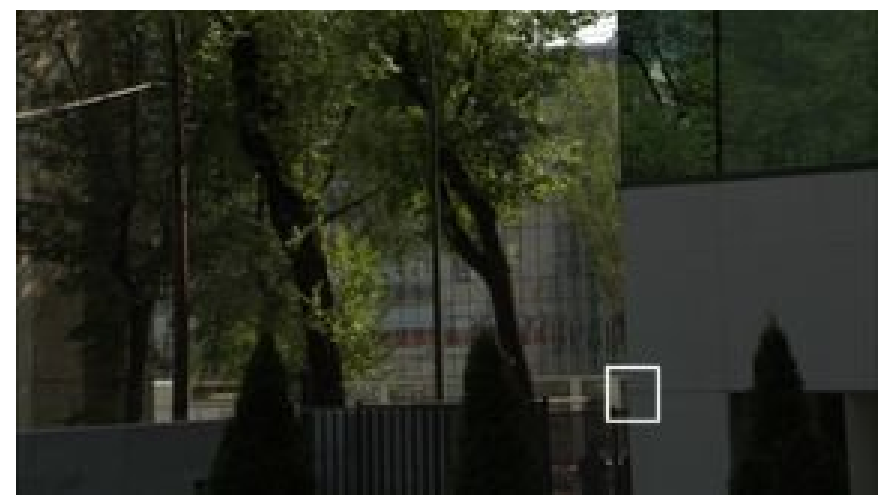

Figure 4: Image taken outside in bright light, with sharp focus containing more high frequency detail. Box shows area used in Figure 5.

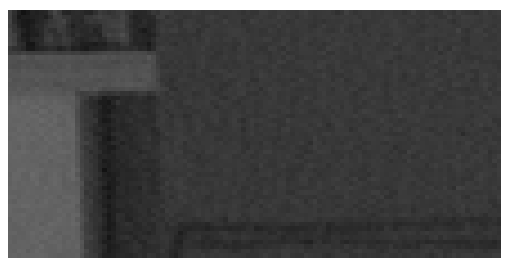

(a) Original

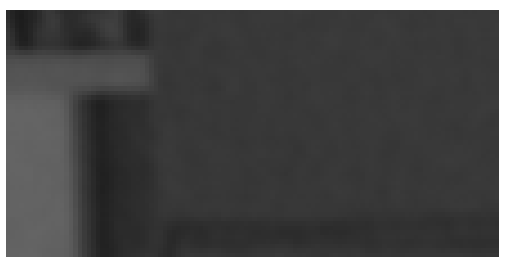

(c) B-spline

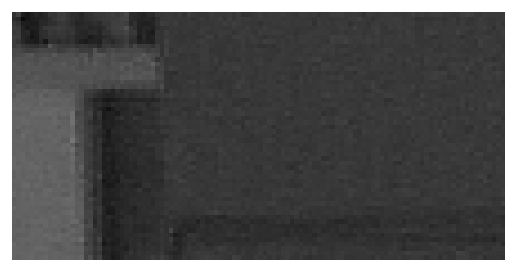

(b) Our algorithm

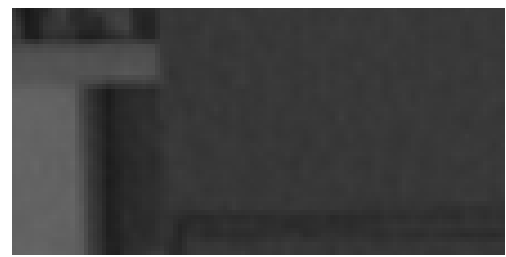

(d) Bilinear

Figure 5: Samples taken from the frame shown in Figure 4. The algorithm convincingly synthesises speckle on the concrete wall but leaves the white wall in the background speckle free.

strength of these frequency components. One of the quality parameters produced by this metric is harmonic loss blur index, which we used to measure the degree of blurriness on our images in this paper. Because blur index is a distortion metric, higher value of the index on an image implies that the image contains more blurring artifacts.

In addition to the above metrics, we also use another quality metric that is sensitive to structural distortions. Blurriness in the expanded images may appear as a result of edge widening as well as change of contrast. The universal quality index (UQI) [9], which is a special case of structural similarity (SSIM) index, has the ability to measure such structural distortions due to image expansion. This metric is significantly different from the traditional PSNR/WPSNR since the latter attempt to quantify the visibility of errors between the degraded images and the original image, whilst UQI models any distortions as a combination of three different factors: loss of correlation, luminance distortion, and 


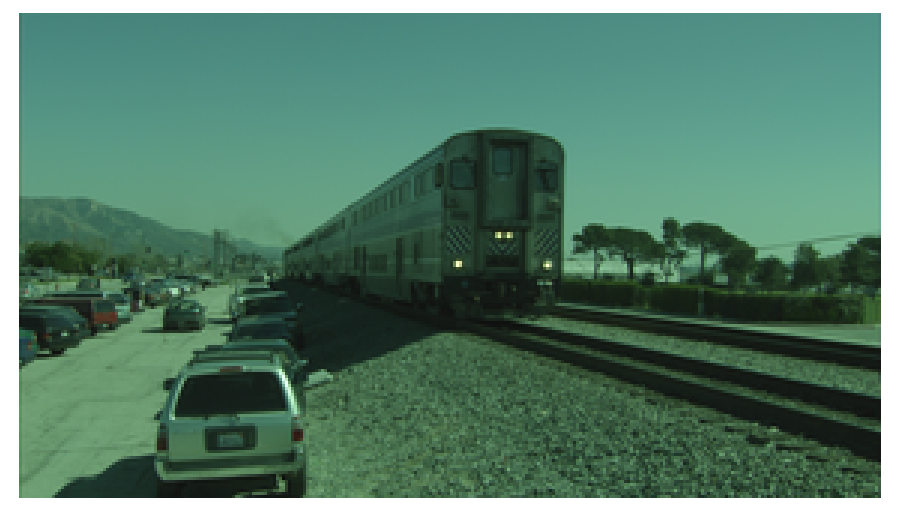

Figure 6: Image from ‘train' sequence. The details are shown in Figure 7.

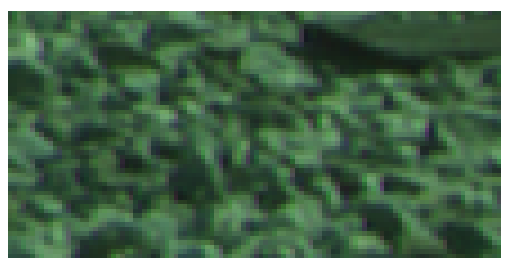

(a) Original

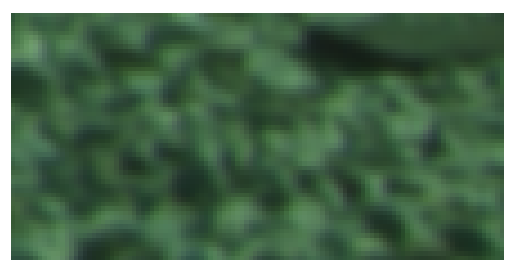

(c) B-spline

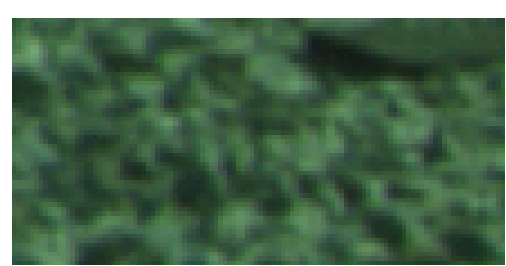

(b) Our algorithm

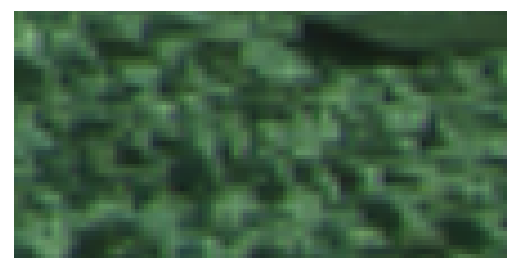

(d) Bilinear

Figure 7: Detail samples from 'train' sequence in Figure 6.

contrast distortion. This metric has a dynamic range of $[-1,1]$, with 1 is the best value (correspond to highest quality).

It is subjectively apparent that our algorithm has regenerated plausible image detail that was irretrievable when using the B-spline and bilinear interpolation approaches (Figures 3, 5, and 7). The down-sampling suppressed visual information which only our algorithm could recover based on its knowledge of statistical co-occurrence of low and high frequency image content. It was also observed that VQ-based enhancement method was better than conventional method (e.g. B-spline) since the latter introduces more blurriness to the processed images. The above mentioned figures show the degree of the blurriness of details from these sequences which have been enhanced by three different methods (our proposed VQ-based, bilinear, and B-spline).

Objective comparison of our algorithm with bilinear and B-spline interpolations for image expansion are shown in Table 1. As an example, Figure 7, which was taken from the 'train' sequence and excluded during the training stage, is used for the computation of 


\begin{tabular}{|c|c|c|c|}
\hline Objective quality metrics & Proposed method & Bilinear & B-Spline \\
\hline \hline PSNR [7] & $36.9 \mathrm{~dB}$ & $28.4 \mathrm{~dB}$ & $36.6 \mathrm{~dB}$ \\
\hline WPSNR [8] & $44.8 \mathrm{~dB}$ & $39.2 \mathrm{~dB}$ & $44.5 \mathrm{~dB}$ \\
\hline Blur index [4] & 0.180 & 0.193 & 0.298 \\
\hline UQI [9] & 0.836 & 0.456 & 0.826 \\
\hline
\end{tabular}

Table 1: Objective image quality metrics for the image expansions shown in Figure 7.

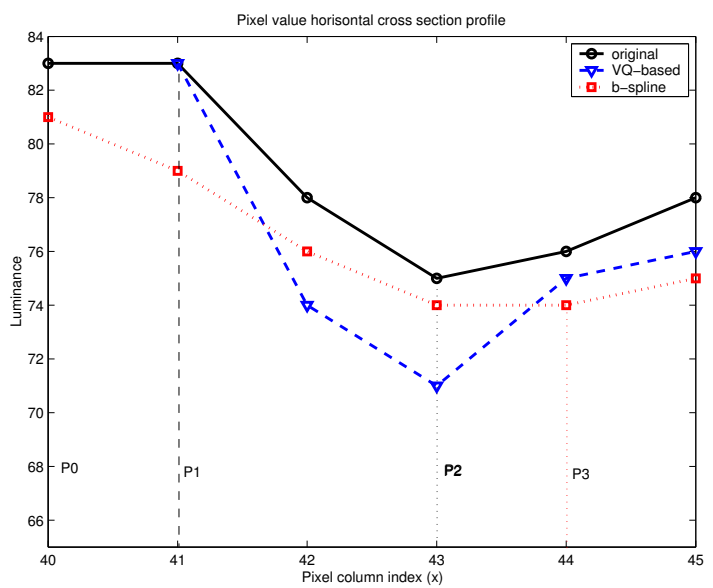

Figure 8: Cross-sectional luminosity profile

these metrics. Our method is superior than the others in terms of the objective measures used in this paper. For example, the blur index values are in favour of our VQ-based method; it shows that the blurriness indices of the B-spline and bilinear enhanced images are generally higher than those of VQ-based, with B-spline enhanced image is the worst in quality (i.e., contains the most blur distortions). The bilinear method, although comparable with our method in terms of blur index, has the most damaged structure as shown by the UQI values.

The blurring effect of expansion method on edges can also be illustrated by observing pixel-value cross-section along a row (or column) in an image. Cross-sectional luminosity profile of the detail images (Figure 7) are shown in Figure 8. In this figure, the local maxima around edges are indicated by the dashed lines $(P 0$ and $P 1)$ and local minima by the dotted lines ( $P 2$ and $P 3)$. The graph shows that the edge in the image expanded by the $\mathrm{B}$-spline method is wider (with an edge width of $P 3-P 0$ ) than those in the original and the VQ-based enhanced images (edge width of $P 2-P 1$ ). It explains that the blur in the traditional expansion method is due to the increased edge width.

\section{Conclusions}

This paper presented a novel approach to image enhancement using a technique which would avoid the known shortcomings of fractal enhancement. We learnt the statistical properties of the co-occurrence of low and high frequency image content and used these 
probability distributions to predict image content during image expansion. Subjective evaluations show that our algorithm outperforms conventional approaches. This is also confirmed by the results of objective evaluations based on several published quality metrics suitable for the application presented here. We hope to continue working on our algorithm which is still in its preliminary stages by: 1) Learning statistical co-occurrence of neighbouring codebook blocks in images; and 2) Mediating the addition of high frequency predicted detail with the energy of the underlying region in the image to prevent prediction of detail in the absence of high frequency information in the original image.

\section{Acknowledgements}

This work is supported by the European Commission under the IP-RACINE project (IST2-511316-IP).

\section{References}

[1] M.F. Barnsley and L.P. Hurd. Fractal image compression. AK Peters, 1993.

[2] N. Chaddha, M. Vishwanath, and PA Chou. Hierarchical vector quantization of perceptually weighted block transforms. Proceedings of the Conference on Data Compression, 1995.

[3] Bernd Girod. What's wrong with mean-squared error? In A. B. Watson, editor, Digital Images and Human Vision, pages 207-220. 1993.

[4] Irwan Prasetya Gunawan and Mohammed Ghanbari. Image quality assessment based on harmonics gain/loss information. In Proceedings of ICIP'05 (IEEE International Conference on Image Processing), volume 1, pages 429-432, Genoa, Italia, September 11-14 2005.

[5] J.R. Levine, T. Mason, and D. Brown. lex \& yacc. O'Reilly \& Associates, Inc. Sebastopol, CA, USA, 1992.

[6] Y. Linde, A. Buzo, and R. Gray. An Algorithm for Vector Quantizer Design. IEEE Transactions on Communications, 28(1):84-95, 1980.

[7] William K. Pratt. Digital Image Processing. John Wiley and Sons, 1978.

[8] Sviatoslav Voloshynovskiy, Alexander Herrigel, Nazanin Baumgärtner, and Thierry Pun. A stochastic approach to content adaptive digital image watermarking. In International Workshop on Information Hiding, volume LNCS 1768 of Lecture Notes in Computer Science, pages 212-236, Dresden, Germany, 29 September -1 October 1999. Springer Verlag.

[9] Zhou Wang, Alan Conrad Bovik, Hamid Rahim Sheikh, and Eero P. Simoncelli. Image quality assessment: From error visibility to structural similarity. IEEE Transaction on Image Processing, 13(4):600-612, April 2004. 